SCIENTIFIC REPORT

\title{
A new form of retinopathy associated with myocardial infarction treated with percutaneous coronary intervention
}

\author{
N Kinoshita, A Kakehashi, T Yasu, T Katayama, M Kuroki, Y Tsurimaki, R Ono, H Yamagami, \\ M Saito, M Kawakami
}

Br J Ophthalmol 2004;88:494-496. doi: 10.1136/bjo.2003.027136

Aim: To report a new form of retinopathy that was observed in patients who had undergone percutaneous coronary intervention ( $\mathrm{PCl}$ ) following acute myocardial infarction (AMI).

Methods: Serial ophthalmological examinations were conducted in 40 patients who underwent $\mathrm{PCl}$. Thirty patients were diagnosed with $\mathrm{AMI}$, and another $10 \mathrm{had}$ stable angina pectoris.

Results: Cotton wool spots developed in 17 (57\%) patients from the group with $\mathrm{AMI}$ undergoing $\mathrm{PCl}(\mathrm{n}=30)$ within 2 months. Of these, $41 \%$ (seven patients) also developed superficial haemorrhages. Retinopathy was most prominent 1-2 months after AMl and then tended to become quiescent afterwards, without treatment.

Conclusion: We have identified a new form of retinopathy in patients with $\mathrm{AMl}$ that spontaneously subsides without treatment.

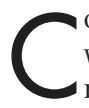
otton wool spots (soft exudates) are transient, small, whitish opacities in the retina and are thought to represent micro-infarctions of small retinal arterioles. In most cases, this retinal change is an indication of systemic vascular disease such as diabetes mellitus or hypertension. ${ }^{12}$ According to Destro and Gragoudas, there can be many underlying causes, including trauma, infection, neoplasia, congenital, haematological, endocrine/gastrointestinal, collagen vascular disease, and vascular disease in general. ${ }^{1} \mathrm{~A}$ general systemic evaluation would yield one of them in as many as $95 \%$ of cases. ${ }^{3}$

Recently, we noticed in one patient that cotton wool spots appeared around the retinal optic disc after percutaneous coronary intervention (PCI) following acute myocardial infarction (AMI). This form of retinopathy subsided gradually during follow up, without treatment. Although there have been a few case reports of retinopathy associated with AMI or $\mathrm{PCI},{ }^{45}$ a prospective case series investigation has not been conducted. These observations prompted us to conduct serial retinal examinations in new patients with AMI to establish a correlation and time course.

\section{MATERIALS AND METHODS}

Serial ophthalmological examinations were conducted in all patients who underwent PCI by percutaneous transluminal coronary angioplasty and stenting at our hospital from May 2001 to March 2002, and gave written informed consent. Thirty patients ( 23 men, 7 women; mean age 59 years; range 39-85) were diagnosed with AMI, and another 10 patients (8 men, 2 women; mean age 69 years; range 57-75) were diagnosed with stable angina pectoris. Another 11 patients were excluded because retinopathy of a known origin was present, including diabetes, hypertension, and renal insufficiency, at the beginning of the study. None of the enrolled patients presented with or developed cardiogenic shock, uncontrolled hypertension or a serious infection throughout the study.

Ophthalmological examinations (visual acuity test and funduscopy) were performed within 3 days of PCI, then at 2 weeks and monthly for 3 months after PCI in the patients who were diagnosed as having AMI, and within 3 days and at 1 month after PCI in the patients with angina.

To further evaluate the retinal microcirculation, fluorescein angiography was performed in three patients with cotton wool spots.

\section{RESULTS}

Cotton wool spots around the optic disc developed in 17 (57\%) of patients from the group with AMI undergoing PCI $(\mathrm{n}=30)$, and superficial haemorrhages were present in $41 \%$ (seven patients) of those 17, during the 3 month period after the onset of AMI. In $71 \%$ (12 of the 17 patients), the retinopathy was bilateral. Fluorescein angiography in three patients showed a non-perfused area consistent with the area of cotton wool spots observed by funduscopy. None of the patients had any objective impairment of visual acuity, although four $(24 \%)$ of the 17 patients with retinopathy complained of blurred vision or metamorphopsia following PCI. In the patients with stable angina, no retinopathy was found at 3 days or 1 month after PCI.

Neither clinical characteristics (sex, age, and the presence of hypertension, diabetes, hyperlipidemia, or smoking) nor laboratory data (peak values of creatine kinase, creatine kinase-MB fraction, $C$ reactive protein and leukocyte density, haematocrit, blood urea nitrogen, creatinine, total cholesterol, triglycerides, and haemoglobin Alc) were significantly different between the groups of patients with and without retinopathy using the Mann-Whitney U test, Student's $t$ test, or multivariate analysis.

Typical fundus and fluorescein angiographic photographs taken at 1 month after AMI are shown in figure 1 . This 46 year old man was diagnosed with AMI and underwent direct angioplasty 3.5 hours after a first episode of severe chest pain. On day 12, cotton wool spots first appeared thinly around the left optic disc. On day 27, they became more noticeable and superficial haemorrhage also appeared (fig 1A). On day 62, the cotton wool spots and superficial haemorrhage began to fade and had nearly disappeared when observed on day 90 .

Figure 2 shows the time course of the ophthalmic manifestations in all patients with AMI. In all patients who

Abbreviations: $\mathrm{AMl}$, acute myocardial infarction; $\mathrm{PCl}$, percutaneous coronary intervention 


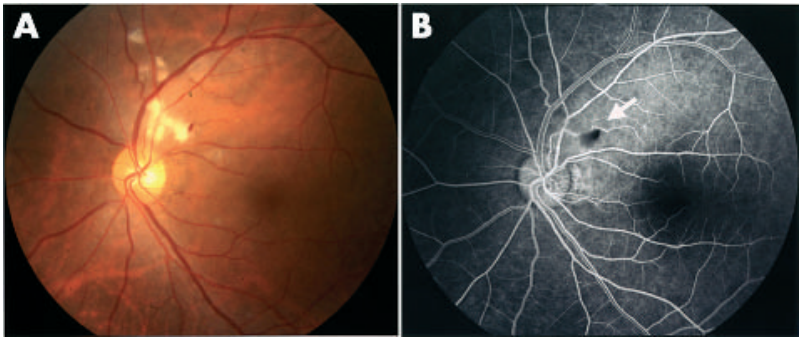

Figure 1 Ophthalmological manifestations 1 month after AMI. Cotton wool spots and superficial haemorrhage are seen adjacent to the optic disc in the left eye (A). (B) Results of fluorescein angiography of the same eye. The arrow indicates a non-perfused area and overlying haemorrhage.

developed retinopathy, it appeared within 2 months after the onset of AMI but was present in most patients at 1 month $(14(82 \%)$ of 17$)$. This retinal change tended to subside within a relatively short period without specific treatment, except for six patients in whom the cotton wool spots persisted for more than 3 months before subsiding.

\section{DISCUSSION}

We report here a new type of retinopathy associated with AMI treated with PCI. Some of the patients recruited in this study had complained of blurred vision after PCI. However, all cases reported here showed spontaneous improvement both in retinal changes and subjective symptoms, without ophthalmological intervention. Therefore, it is important for both ophthalmologists and physicians to be aware of this transient form of retinopathy associated with AMI and PCI, and not hastily perform any further examinations such as fluorescein angiography.

Cotton wool spots, a primary feature of this form of retinopathy, are thought to be related to focal acute retinal ischaemia. ${ }^{12}$ Cotton wool spots occur frequently in diabetic retinopathy and hypertensive retinopathy. However, the retinopathy in this study associated with AMI and PCI can be easily differentiated from these other forms of retinopathy by the absence of other changes that commonly coexist with cotton wool spots. In diabetic retinopathy, the most common aetiology of cotton wool spots, capillary microaneurysms, dot and blot haemorrhages, and hard exudates usually precede their appearance. Although some patients in this study had diabetes, it was not difficult to differentiate this new form of retinopathy associated with AMI from typical diabetic retinopathy that should have the above described characteristics. Hypertensive retinopathy could also be differentiated from the AMI associated retinopathy by the absence of both uncontrolled hypertension and hypertensive retinal arteriolar changes characterised by noticeable narrowing and vasoconstriction.

Acute myocardial infarction is frequently treated with reperfusion therapy. When the occluded coronary arteries are reperfused, ischaemia/reperfusion injury occurs not only in the heart but also in remote organs as a consequence of the release of proinflammatory cytokines, activation of leukocytes, increase in free radicals and adhesion molecules, and endothelial dysfunction. ${ }^{6-11}$ These consequences may be a cause of the unusual form of retinopathy in the present study, since fluorescein angiography demonstrated that the cotton wool spots occurred in non-perfused areas of the retinal microvasculature.

Because the therapeutic benefits of PCI in AMI have been well established, all newly diagnosed AMI patients in our

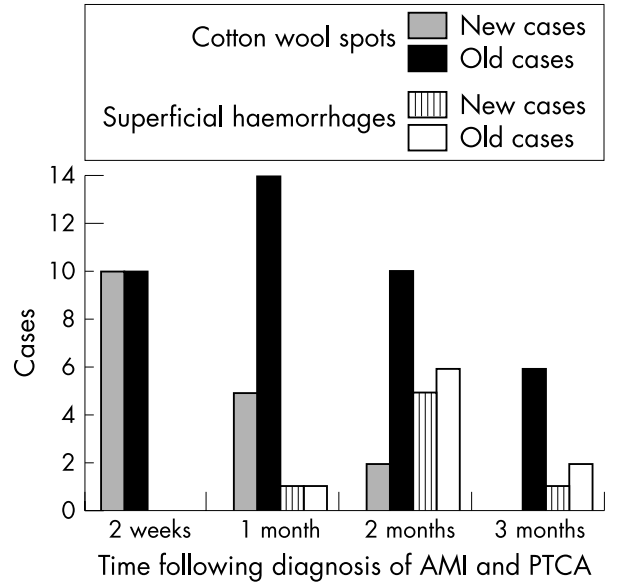

Figure 2 Time course of ophthalmic manifestations. Cotton wool spots were most frequently observed at 1 month and superficial haemorrhages at 2 months.

hospital underwent PCI, and thus we could not determine whether AMI patients who do not undergo PCI will also develop this form of retinopathy. However, none of the patients with stable angina who underwent PCI developed retinopathy. A previous study aiming to examine the adverse effects of thrombolytic therapy on the retina identified cotton wool spots and/or haemorrhage in only three of 60 acute cardiac chest pain patients treated with or without thrombolytic therapy, ${ }^{12}$ however retinal changes were not followed long enough after the onset of chest pain to detect the retinopathy reported here. In addition to the above study, there have been a few case reports of retinopathies associated with AMI and PCI. ${ }^{4}$ The pathogenesis has been mainly attributed to retinal artery occlusion by microembolism of cholesterol crystals or a microthrombus. The retinopathy reported in our study appears to be a different clinical entity characterised by the transient appearance of cotton wool spots and the lack of any signs of cholesterol crystal embolisation or thrombus in the retinal artery ${ }^{4}$ or other organs including the brain. ${ }^{13-16}$

This new form of transient retinopathy should be considered in patients undergoing PCI after a recent episode of AMI, and carefully differentiated from other forms of cotton wool spots, in order to avoid unnecessary invasive examinations.

\section{ACKNOWLEDGEMENTS}

We thank K Kasono, MD, H Ueba, MD, H Tamemoto, MD, K Namai, MD, and S Ishikawa, MD, for their helpful suggestions; M Takahashi, A Yamaguchi, T Shibuya, K Reisui, and S Yamazaki for assistance with the ophthalmological examinations; and Michael A Yamin, $\mathrm{PhD}$, for editing the manuscript.

\section{Authors' affiliations}

N Kinoshita, A Kakehashi, Y Tsurimaki, R Ono, H Yamagami, Department of Ophthalmology, Omiya Medical Center, Jichi Medical School, Saitama, Japan

T Yasu, T Katayama, M Kuroki, M Saito, M Kawakami, Department of Comprehensive Medicine I, Omiya Medical Center, Jichi Medical School, Saitama, Japan

Correspondence to: Dr M Kawakami, Department of Comprehensive Medicine I, Omiya Medical Center, Jichi Medical School, 1-847 Amanuma, Saitama, Saitama 330-8503, Japan;

nobukawa@omiya.jichi.ac.jp

Accepted for publication 1 September 2003 


\section{REFERENCES}

1 Destro M, Gragoudas ES. Arterial occlusions. In: Albert DM, Jakobiec FA, eds. Principle and practice of ophthalmology. Philadelphia: WB Saunders Company, 1994:727-8.

2 Brown GC. Retinal arterial obstructive disease. In: Ryan SJ, ed. Retina, 2nd ed. St Louis, MO: Mosby-Year Book, Inc, 1994:1373-5.

3 Brown GC, Brown MM, Hiller T, et al. Cotton wool spots. Retina 1985:5:206-14.

4 Teitelbaum BA. Asymptomatic unilateral microembolic retinopathy secondary to percutaneous transluminal coronary angioplasty. J Am Optom Assoc 1999;70:587-92

5 Bucci FA Jr, Dimitsopulos TM, Krohel GB. Branch retinal artery occlusion secondary to percutaneous transluminal coronary angioplasty. Br J Ophthalmol 1989;73:309-10.

6 Carden DL, Granger DN. Pathophysiology of ischaemia-reperfusion injury. J Pathol 2000;190:255-66.

7 Abe $Y$, Kawakami M, Kuroki $M$, et al. Transient rise in serum interleukin 8 concentration during acute myocardial infarction. Br Heart J 1993;70:132-4.

8 Neumann F, Ott I, Gawaz M, et al. Cardiac release of cytokines and inflammatory responses in acute myocardial infarction. Circulation 1995;92:748-55
9 Ambrosio G, Tritto I. Reperfusion injury: experimental evidence and clinical implications. Am Heart J 1999; 138:S69-75.

10 Kharbanda RK, Peters M, Walton B, et al. Ischemic preconditioning prevents endothelial injury and systemic neutrophil activation during ischemiareperfusion in humans in vivo. Circulation 2001;103:1624-30.

11 Yasu T, lkeda N, Ishizuka N, et al. Nicorandil and leukocyte activation. J Cardiovasc Pharmacol 2002;40:684-92.

12 Sleightholm M, Wanklyn P, Kearney M. Haemorrhagic retinopathy in patients admitted with acute cardiac chest pain. Eur Heart J 1996;17:481.

13 Bladin CF, Bingham L, Grigg L, et al. Transcranial doppler detection of microemboli during percutaneous transluminal coronary angioplasty. Stroke 1998;29:2367-70.

14 Leclercq F, Kassnasrallah S, Cesari JB, et al. Transcranial doppler detection of cerebral microemboli during left heart catheterization. Cerebrovasc Dis 2001;12:59-65.

15 Eggenberger ER, Desai NP, Kaufman DI, et al. Internuclear ophthalmoplegia after coronary artery catheterization and percutaneous transluminal coronary balloon angioplasty. J Neuroophthalmol 2000;20:123-6.

16 Drummond GT, Weubbolt G. Bilateral ophthalmoplegia during percutaneous transluminal coronary angioplasty. Can J Ophthalmol 1990;25:152-5 